\title{
1 abmR: an R package for agent-based model analysis 2 of large-scale movements across taxa
}

6 Benjamin Gochanour ${ }^{1,2, *}$, Javier Fernández-López ${ }^{3}$, Andrea Contina ${ }^{4}$

$7 \quad{ }^{1}$ Corix Plains Institute, University of Oklahoma, Five Partners Place, 201 Stephenson Parkway, Norman, OK 873019, USA

$9{ }^{2}$ Oklahoma Biological Survey, University of Oklahoma, 111 East Chesapeake Street, SC Building 134, 10 Norman, OK 73019, USA.

113 University of Massachusetts Boston, Department of Biology, Integrated Sciences Complex, 100 Morrissey

12 Blvd., Boston, MA 02125

134 University of Colorado Denver, Department of Integrative Biology, Science Building 2074, Denver, CO 14 80217, USA

*Corresponding author

17 *E-mail: gocha001@umn.edu

Benjamin Gochanour ORCID: 0000-0002-0950-1739 


\section{Abstract}

1. Agent-based modeling (ABM) shows promise for animal movement studies. However, a robust, open-source, and spatially explicit ABM coding platform is currently lacking.

2. We present abmR, an $\mathrm{R}$ package for conducting continental-scale ABM simulations across animal taxa. The package features two movement functions, each of which relies on the Ornstein-Uhlenbeck (OU) model.

3. The theoretical background for abmR is discussed and the main functionalities are illustrated using two example populations.

4. Potential future additions to this open-source package may include the ability to specify multiple environmental variables or to model interactions between agents. Additionally, updates may offer opportunities for disease ecology and integration with other R movement modeling packages.

\section{Keywords:}

Animal migration; R programming; computer simulations; ecology

Abbreviated Title: agent-based models in R 


\section{INTRODUCTION}

52 Animal movement is a complex behavioral trait that affects the survival of populations and species across taxa (Berg, 1983; Dingle, 2014). Long- and short-distance movements can be predictable, allowing populations to take advantage of seasonal food resources (e.g., migration), or more opportunistic, such as in the case of dispersal behaviors aimed at avoiding predators or finding potential mates (Giuggioli \& Bartumeus, 2010). Thus, wild animals make decisions often based on environmental cues that lead to movement patterns characteristic of different populations across the landscape (Nathan et al., 2008; Dodge et al., 2014). However, obtaining a comprehensive understanding of large-scale animal movement behavior and population occurrence under climate change scenarios or habitat loss has proven to be a challenge (Araujo \& Guisan, 2006). Moreover, while the research toolbox in movement ecology studies has seen a considerable expansion over the last two decades due to technological advancements of the tracking devices and molecular markers (Cushman and Lewis, 2010; Williams et al., 2020), the limitation of scaling up individual data to populationlevel inferences is still a substantial obstacle (Hawkes, 2009; but see Holdo \& Roach, 2013). A promising research approach that may overcome the limitations of wildlife movement studies hindered by small sample sizes is represented by computer simulations within an Agent-based Modeling (ABM) framework (Tang \& Bennett, 2010; Bridge et al., 2017).

The core principle of $\mathrm{ABM}$ is to simulate a set of entities, called agents, which are defined by intrinsic properties as well as behavioral rules governing their interactions with the environment (Grimm \& Railsback, 2013). That is, agents are described by their inherent attributes while dynamically interacting with external conditions such as the co-occurrence of other agents and/or changing features of their environmental setting . Thus, ABM has found applications in many study areas including biology, disease risk, social sciences, and 
economics (Polhill et al., 2008; Grimm \& Railsback, 2013; Kilmek et al., 2015; Willem et al., 2017) with the unifying goal of investigating and predicting the dynamics of complex systems (Grimm et al., 2005). In particular, wildlife studies have adopted the ABM approach to simulate population growth, reproduction, mortality rate, energy budget, and migration ecology, just to cite a few (Brown \& Robinson, 2006; Lustig et al., 2019; Aurbach et al., 2020; Goldstein et al., 2021). However, we currently lack a robust and spatially explicit ABM coding platform for the implementation of large-scale animal movement investigations (but see Thiele et al. (2012) or Chubaty and McIntire (2021)). Here we present a novel ABM framework in the R programming language for applications in animal behavior and movement ecology broadly defined.

\section{PACKAGE OVERVIEW}

abmR allows for both computation and visualization of agent movement trajectories through a set of behavioral rules based on environmental parameters. The two movement functions, moveSIM and energySIM, provide the central functionality of the package, allowing the user to run simulations using an Ornstein-Uhlenbeck movement model (Uhlenbeck \& Ornstein, 1930; hereafter OU). Additional functions provide a suite of visualization and data summarization tools intended to reduce the effort needed to go from results to presentationready figures and tables (Table 1). The package is currently available as a Github repository (https://github.com/bgoch5/abmR), but has been submitted to the Comprehensive R Archive Network (CRAN) to facilitate broader access and usage. 
96

\section{Usage}

\begin{tabular}{ll}
\hline moveSIM & Runs agent-based model movement simulations based on environmental data \\
moveVIZ & Creates a plot or table of moveSIM () results \\
energySIM & Runs agent-based model movement and energy budget simulations based on environmental data \\
energyVIZ & Creates a plot or table of energySIM () results \\
tidy_results & Prints results from moveSIM () or energySIM () in an easier-to-read table \\
get_ex_data & Downloads data that is used in examples in vignette and documentation \\
as.species & Creates object of class 'species' for input into moveSIM () or energySIM () \\
\hline
\end{tabular}

Table 1. Functions contained in the abmR package (v. 1.0.1). For more complete function descriptions, consult the abmR manual.

Both movement functions used by abmR rely on the same OU model approach summarized below. Given current agent location $\left(\mathrm{x}_{\mathrm{t}}, \mathrm{y}_{\mathrm{t}}\right)$, agent location at the subsequent timestep $\left(\mathrm{x}_{\mathrm{t}+1}\right.$, $\left.\mathrm{y}_{\mathrm{t}+1}\right)$ is modeled according to the following equations:

$$
\begin{aligned}
& x_{t+1}=x_{t}+\sigma * Z+\phi_{x} *\left(\omega_{x}-x_{t}\right) \\
& y_{t+1}=y_{t}+\sigma * Z+\phi_{y} *\left(\omega_{y}-y_{t}\right)
\end{aligned}
$$

Here, $\sigma$ is the randomness parameter from the Brownian motion process that serves as a multiplier on the error term $\mathrm{Z}$, a single random number drawn from the $\operatorname{Normal}(0,1)$ distribution. In addition, $\phi_{x}$ and $\phi_{y}$ are movement motivation or attraction strength for the OU process in the longitude and latitude coordinates, respectively, while $\omega_{x}$ and $\omega_{y}$ are optimal $\mathrm{x}$ (longitude) and $\mathrm{y}$ (latitude) coordinates, respectively. It is assumed that the origin point ( $\mathrm{x}_{1}$, $\left.\mathrm{y}_{1}\right)$ is known. The OU model given in (1) and (2) performs similarly to a spring-coil. Greater distance from optimal coordinates $\omega_{x}$ and $\omega_{y}$ acts like a compressed spring to propel distant agents towards $\omega_{x}$ and $\omega_{y}$. On the other hand, agents closer to $\omega_{x}$ and $\omega_{y}$ will travel a shorter distance on that timestep. However, the amount of movement also depends on $\phi_{x}$ and $\phi_{y}$, because these motivations serve as a multiplier on $\left(\omega_{x}-x_{t}\right)$ and $\left(\omega_{y}-y_{t}\right)$, respectively (Eqns. 1 and 2). 


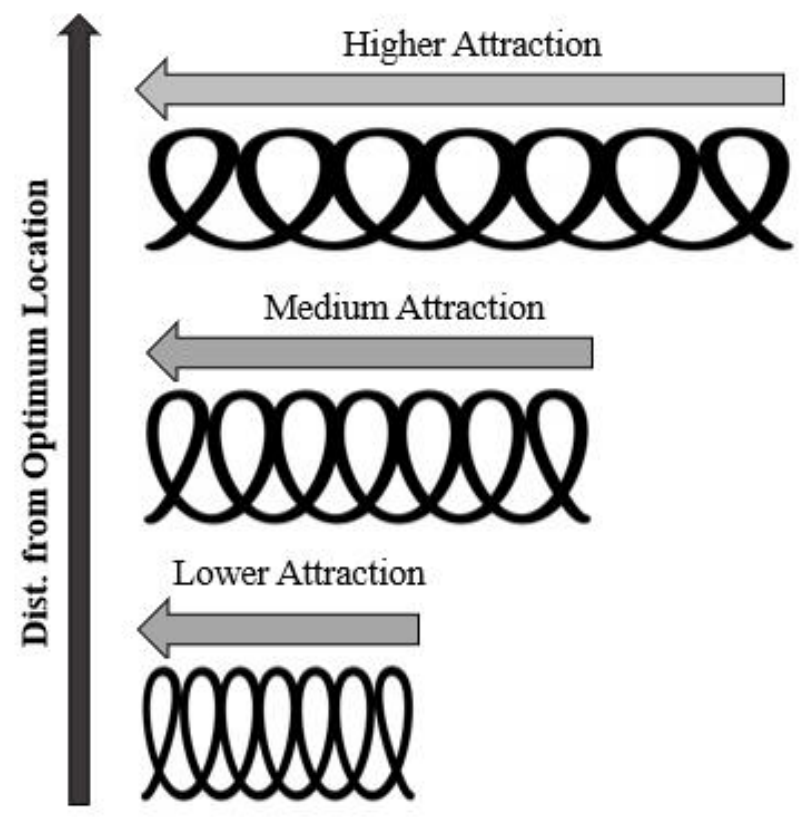

Figure 1. The OU model given in (1) and (2) performs like a spring-coil: agents further from their target location experience higher attraction (and travel further), while agents closer to their destination experience lesser attraction (and travel less far).

Both movement functions allow the user to optionally specify two morphological parameters. $\phi_{y}$ from user-specified motivations $\phi_{x 0}$ and $\phi_{y 0}$.

Here, $a, \mu_{a}$, and $S_{a}$ represent the observed value, population mean, and population standard deviation for the first morphological parameter, respectively, while $b, \mu_{b}$, and $S_{b}$ represent constant 0.1 depends upon the hypothesized effect of each morphological parameter on agent movement. If increasing values of the parameter lead to increasing motivation, a positive sign 
should be used, and if increasing values of the parameter lead to decreasing motivation, a negative sign should be used. For example, if one hypothesizes that increasing mass leads to less movement motivation, a negative sign should be used for this parameter. The biological basis for including morphological parameters as a multiplier on motivation relies on previous research showing that individual and population intrinsic morphological characteristics affect migration speed, distance, and timing in many species (Alerstam, 1993; Hedenström, 2008).

While the two movement functions are distinct (see below), each follows the same basic two steps. The first, large-scale searching, is illustrated in Fig. 2. This step finds the 'optimum' location for each agent ( $\omega_{x}$ and $\omega_{y}$ coordinates from Eqns. (1) and (2)), that is, the geographic point with the optimal environmental raster value in a semicircular search region depending on movement orientation. For moveS IM, this optimal raster value is supplied directly, while for energySIM it is the average of the lower and upper bounds of a user-specified optimum range. The optimum value or range of values specified depends on the modeling scenario and the type of environmental raster that is used (e.g., vegetation, temperature, etc.).

Agents will move toward the selected optimum location. However, if the attraction strength ( $\phi_{x}$ and $\phi_{y}$ in Eqns. (1) and (2)) is less than 1, agents will have a 'target' location short of the optimal location. Agents will move towards this target location with some error, which is generated by sampling from the normal distribution and multiplying by $\sigma$, as specified by the user (In Fig. 2, $\sigma$ is 1). Because the support of the normal distribution consists of all real numbers, large deviations from the 'target' point are possible. However, because the normal distribution has low density at the extreme tails, outcomes are most likely to fall within a certain rectangle of the target, as illustrated in Fig. 2. This first step corresponds to the OU model of Eqns. (1) and (2). 


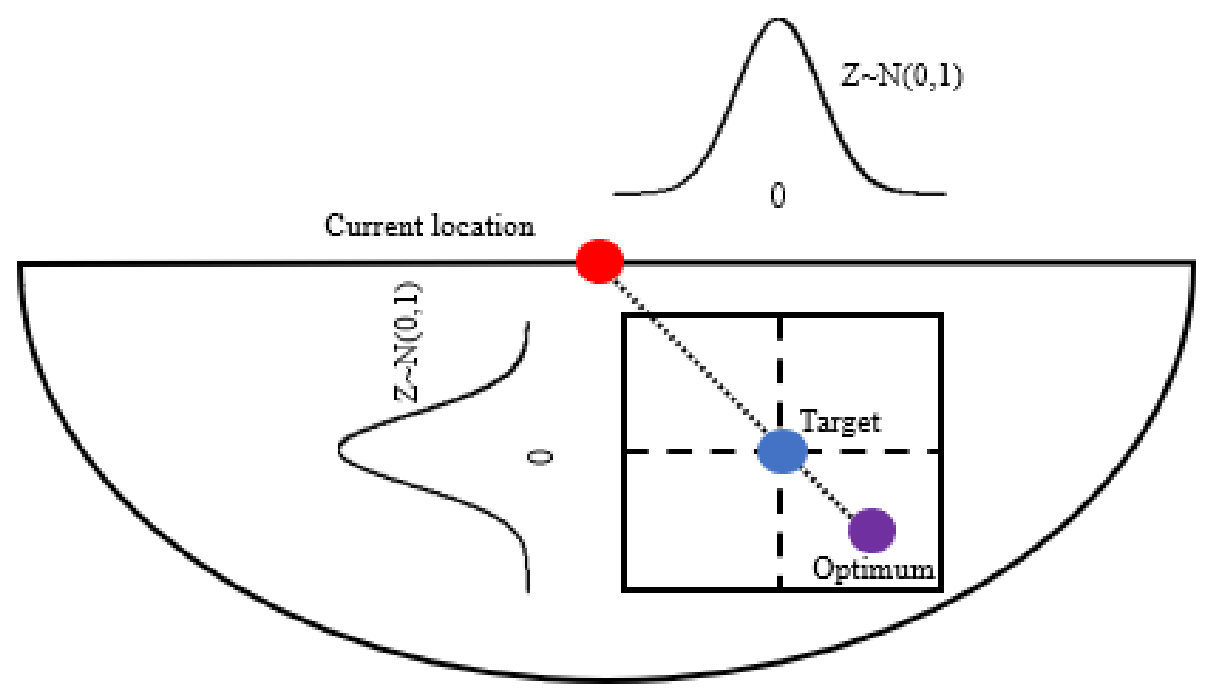

153 Figure 2. Illustration of large-scale searching specified by the OU model of Eqns. (1) and (2). Agents 154 find an 'optimum' location within the semi-circular search region and then a 'target' location that lies on the line between the 'current location' and the 'optimum' location. If there is a tie between multiple potential 'optimum' cells, one is randomly selected from the list of tied cells to serve as the optimum. Random error is added by sampling from a $\mathrm{Z} \sim \mathrm{N}(0,1)$ distribution. Here, $\sigma=1$ and $\phi_{\mathrm{x}}=$ $\phi_{\mathrm{y}}<1$, where $\sigma$ is the multiplier on the random error and $\phi_{\mathrm{x}}$ and $\phi_{\mathrm{y}}$ are the motivations in the $\mathrm{x}$ and $\mathrm{y}$ directions, respectively. Bounding box represents the most probable samples from the $\mathrm{N}(0,1)$ distribution.

The second step is small-scale searching. Here, agents select the 'best' of the 8 neighboring cells (queen's case or Moore neighborhood) after performing step 1, discussed above and in Fig 2. Again, 'best' here means the cell with environmental raster value closest to the agent's user-defined optimum range. These two steps are then repeated for each timestep until the agent dies or proceeds through all timesteps. For the moveS IM function, agent death occurs when agents fail to achieve suitable environmental raster values for more than a userspecified number of consecutive timesteps. Here, what constitutes a 'suitable' cell is determined by the optimum value and an allowable deviation proportion, both also specified 
by the user. For the energySIM function, agent death occurs when energy reaches zero. For both functions, users may choose to disable agent mortality. In the following subsections, we

171 present the differences between moveSIM and energyS IM functions and their underlying 172 algorithms.

\section{2.1. Simulation function: moveSIM}

174 The function moveS IM runs an OU movement simulation based on environmental conditions

175 provided by the user (e.g., raster), optionally including agent mortality and adjusted

176 motivation according to user-specified morphological parameters. The function operates

177 according to the following algorithm. Here, terms in italics are moveS IM function arguments 178 (see Table 2). 


\begin{tabular}{|c|c|c|}
\hline Argument & Function & Usage \\
\hline replicates & $\mathrm{M}, \mathrm{E}$ & number of agents to model \\
\hline days & $\mathrm{M}, \mathrm{E}$ & number of timesteps \\
\hline modeled_species & $\mathrm{M}, \mathrm{E}$ & Species object from as.species () \\
\hline env_rast & $\mathrm{M}, \mathrm{E}$ & Environmental raster \\
\hline optimum & M & Optimal environmental value \\
\hline optimum_lo & $\mathrm{E}$ & Lowest optimum environmental value \\
\hline optimum_hi & $\mathrm{E}$ & Highest optimum environmental value \\
\hline dest_x & $\mathrm{M}, \mathrm{E}$ & Destination Longitude \\
\hline dest_y & $\mathrm{M}, \mathrm{E}$ & Destination Latitude \\
\hline mot_x & $\mathrm{M}, \mathrm{E}$ & Motivation ( $\mathrm{x}$ direction) \\
\hline mot_y & $\mathrm{M}, \mathrm{E}$ & Motivation (y direction) \\
\hline search_radius & $\mathrm{M}, \mathrm{E}$ & Radius of semi-circular search region $(\mathrm{km})$ \\
\hline direction & $\mathrm{M}, \mathrm{E}$ & Movement direction: N, S, E, W, or R (Random) \\
\hline sigma & $\mathrm{M}, \mathrm{E}$ & Randomness parameter \\
\hline mortality & $\mathrm{M}, \mathrm{E}$ & Incorporate agent mortality? $\mathrm{T}$ or $\mathrm{F}$ \\
\hline fail_thresh & $\mathrm{M}$ & Deviation from optimum constituting failure \\
\hline n_failures & M & Allowable number of failures before agent death \\
\hline init_energy & $\mathrm{E}$ & Initial energy \\
\hline energy_adj & $\mathrm{E}$ & Energy gain/penalty vector \\
\hline single_rast & $\mathrm{M}, \mathrm{E}$ & Using a single-layer raster? $\mathrm{T}$ or $\mathrm{F}$ \\
\hline write_results & $\mathrm{M}, \mathrm{E}$ & Save results as a csv? $\mathrm{T}$ or $\mathrm{F}$ \\
\hline $\mathrm{x}$ & $\mathrm{S}$ & Origin longitude \\
\hline $\mathrm{y}$ & $\mathrm{S}$ & Origin latitude \\
\hline morphparl & $\mathrm{S}$ & Morphological parameter num. 1 observed value \\
\hline morphparlmean & $\mathrm{S}$ & Morphological parameter num. 1 mean \\
\hline morphparlsd & $\mathrm{S}$ & Morphological parameter num. 1 SD \\
\hline morphparlsign & $\mathrm{S}$ & Morphological parameter num. 1 sign \\
\hline morphpar2 & $\mathrm{S}$ & Morphological parameter num. 2 observed value \\
\hline morphpar2mean & $\mathrm{S}$ & Morphological parameter num. 2 mean \\
\hline morphpar2sd & $\mathrm{S}$ & Morphological parameter num. 2 SD \\
\hline morphpar2sign & $\mathrm{S}$ & Morphological parameter num. 2 sign \\
\hline
\end{tabular}

181 Table 2. List of arguments used in moveSIM (M), energySIM (E), or as.species (S). T: True,

182 F: False, SD: standard deviation. In text, these arguments are presented in italics. For a more complete

183 list of argument descriptions, see the abmR documentation.

184 The following algorithm applies when the argument direction is 'N', 'S', 'E', or 'W'. For random movement $($ direction $=$ ' $R$ ') agents simply select a random point from a circle of 
radius search_radius for each timestep (Step 4). Here, let env_rast $\left(x_{t+1}, y_{t+1}\right)$ be the value of env_rast at the point $\left(x_{t+1}, y_{t+1}\right)$. The core algorithm shown here assumes that env_rast contains no undefined (N/A) grid cells. $\phi_{x}$ and $\phi_{y}$ according to (3) and (4), respectively. If not, set $\phi_{x}=\phi_{x 0}$ and $\phi_{y}=\phi_{y 0}$, where $\phi_{x 0}$ and $\phi_{y 0}$ are $m o t_{-} x$ and $m o t_{-} y$, respectively.

3. Set failures $=0$

4. For day $t$ in 1:(days-1)

a. Create a search area defined as a semicircle of radius search_radius facing direction and centered at $\left(x_{t}, y_{t}\right)$

b. Determine $\left(\omega_{x}, \omega_{y}\right)$ as location within the search area with env_rast cell value closest to optimum. Draw a random sample $(\mathrm{n}=1)$ in case of ties.

i. If $($ dest_x, dest_x $)$ in search area, set $\left(\omega_{x}, \omega_{y}\right)=\left(d e s t \_x\right.$, dest_y $)$

c. Large scale searching: determine $\left(x_{t+1}, y_{t+1}\right)_{0}$ according to (1) and (2).

d. Small scale searching: determine $\left(x_{t+1}, y_{t+1}\right)$ by selecting location within eight neighboring cells (queen's case) of $\left(x_{t+1}, y_{t+1}\right)_{0}$ with the cell value closest to optimum. Draw a random sample $(\mathrm{n}=1)$ in case of ties. failures $=$ failures +1 . If not, set failures $=0$. 
5. Return dataframe with days rows and 2 columns movement track data.

6. Repeat (3)-(5) replicates times.

\subsection{Simulation function: energySIM}

The function energyS IM builds on moveS IM by allowing for dynamic agent energy levels that are affected by the quality of environmental values achieved. These initial user-defined energy levels then serve as a driver of mortality and movement distance per timestep. It

The following algorithm applies when the argument direction is ' $\mathrm{N}$ ', ' $\mathrm{S}$ ', ' $\mathrm{E}$ ', or ' $\mathrm{W}$ '. For random movement $($ direction $=$ ' $R$ ') agents simply select a random point from a circle of radius search_radius for each timestep (Step 5). Here, let env_rast $\left(x_{t+1}, y_{t+1}\right)$ be the value of env_rast at the point $\left(x_{t+1}, y_{t+1}\right)$. The core algorithm shown here assumes that env_rast $\phi_{y 0}$, where $\phi_{x 0}$ and $\phi_{y 0}$ are mot_x and mot_y, respectively.

3. Specify $\left(\mathrm{x}_{1}, \mathrm{y}_{1}\right)$ using $x$ and $y$ contained in modeled_species.

4. Set energy $=$ init_energy

5. For day $\mathrm{t}$ in $1:($ days -1$)$ 
a. If mortality $=$ True, update search_radius as search_radius $=$ search_radius $*($ energy/init_energy).

b. Create a search area defined as a semicircle of radius search_radius facing direction and centered at $\left(x_{t}, y_{t}\right)$.

c. Determine $\left(\omega_{x}, \omega_{y}\right)$ as location within the search area with env_rast cell value closest to optimum. Draw a random sample $(\mathrm{n}=1)$ in case of ties.

i. If $($ dest_x, dest_x $)$ in search area, set $\left(\omega_{x}, \omega_{y}\right)=\left(d e s t \_x\right.$,

$$
\text { dest_y) }
$$

d. Large scale searching: determine $\left(x_{t+1}, y_{t+1}\right)_{0}$ according to (1) and (2).

e. Small scale searching: determine $\left(x_{t+1}, y_{t+1}\right)$ by selecting location within eight neighboring cells (queen's case) of $\left(x_{t+1}, y_{t+1}\right)_{0}$ with the ties.

f. If optimum_lo<env_rast $\left(x_{t+1}, y_{t+1}\right)<o p t i m u m \_h i$ update energy $=$ energy+energy_adj[1].

g. Else, compute $\Delta=$ env_rast $\left(x_{t+1}, y_{t+1}\right)$ - optimum. And update energy in the following way:

If $\Delta<0.1 *$ optimum, then energy $=$ energy+energy_adj[2]

energy+energy_adj[3] 
energy+energy_adj[6]

If $0.5 *$ optimum $<\Delta<0.6 *$ optimum, then energy $=$

energy+energy_adj[7]

If $0.6 *$ optimum $<\Delta<0.7 *$ optimum, then energy $=$

energy+energy_adj[8]

If $0.7 *$ optimum $<\Delta<0.8 *$ optimum, then energy $=$

energy+energy_adj[9]

energy+energy_adj[10] through $\left(x_{\text {days }}, y_{\text {days }}\right)$ as N/A and end loop.

6. Return dataframe with days rows and 2 columns movement track data.

\section{EXAMPLE APPLICATIONS}

272 abmR can be used to construct ABM simulations for any desired agent across the globe. In

273 the following example, we demonstrate how energyS IM can be used to compare the

274 movement and the differential energy allocation of two populations of 250 agents each.

275 While we focus on energyS IM in this example, a similar workflow applies for moveS IM. 


\subsection{Comparisons between populations}

277 In this example, both populations are characterized by the same number of replicates, movement timesteps ('days'), the same $\sigma$, and the same environmental data provided by a movements from a different point $\left(105.7^{\circ} \mathrm{W} ; 48.2^{\circ} \mathrm{N}\right)$ situated about 2,800 $\mathrm{km}$ from the origin of Population $2(\mathrm{P} 2)$ agents $\left(142.7^{\circ} \mathrm{W} ; 63.2^{\circ} \mathrm{N}\right)$. Additionally, P1 agents had a

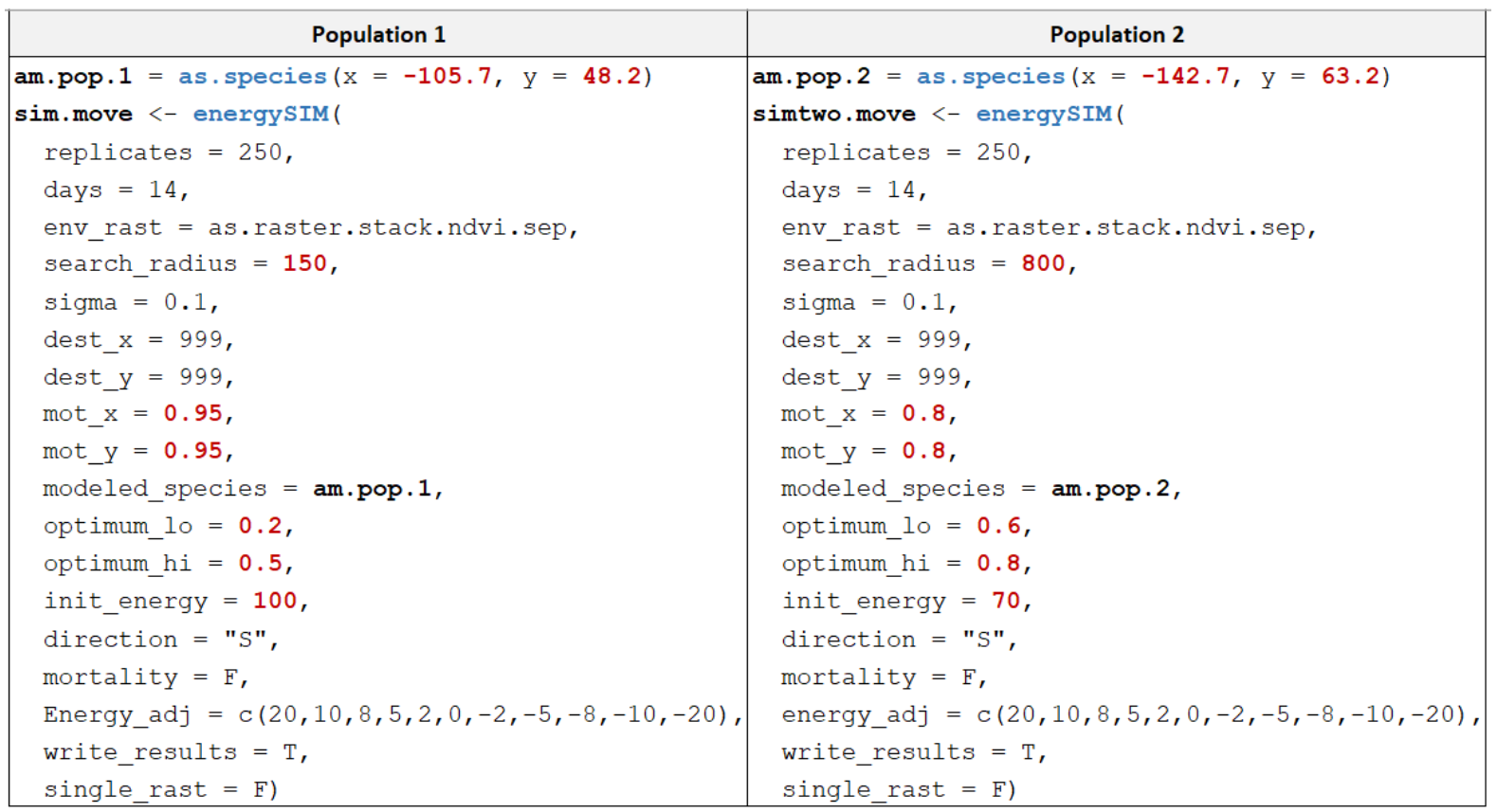

Box 1. R code used for performing the simulations presented in Fig 3. First, as . species is called to initialize two populations with different origin locations (here omitting morphology). Then, 

descriptions, see Table 2 and the package manual.
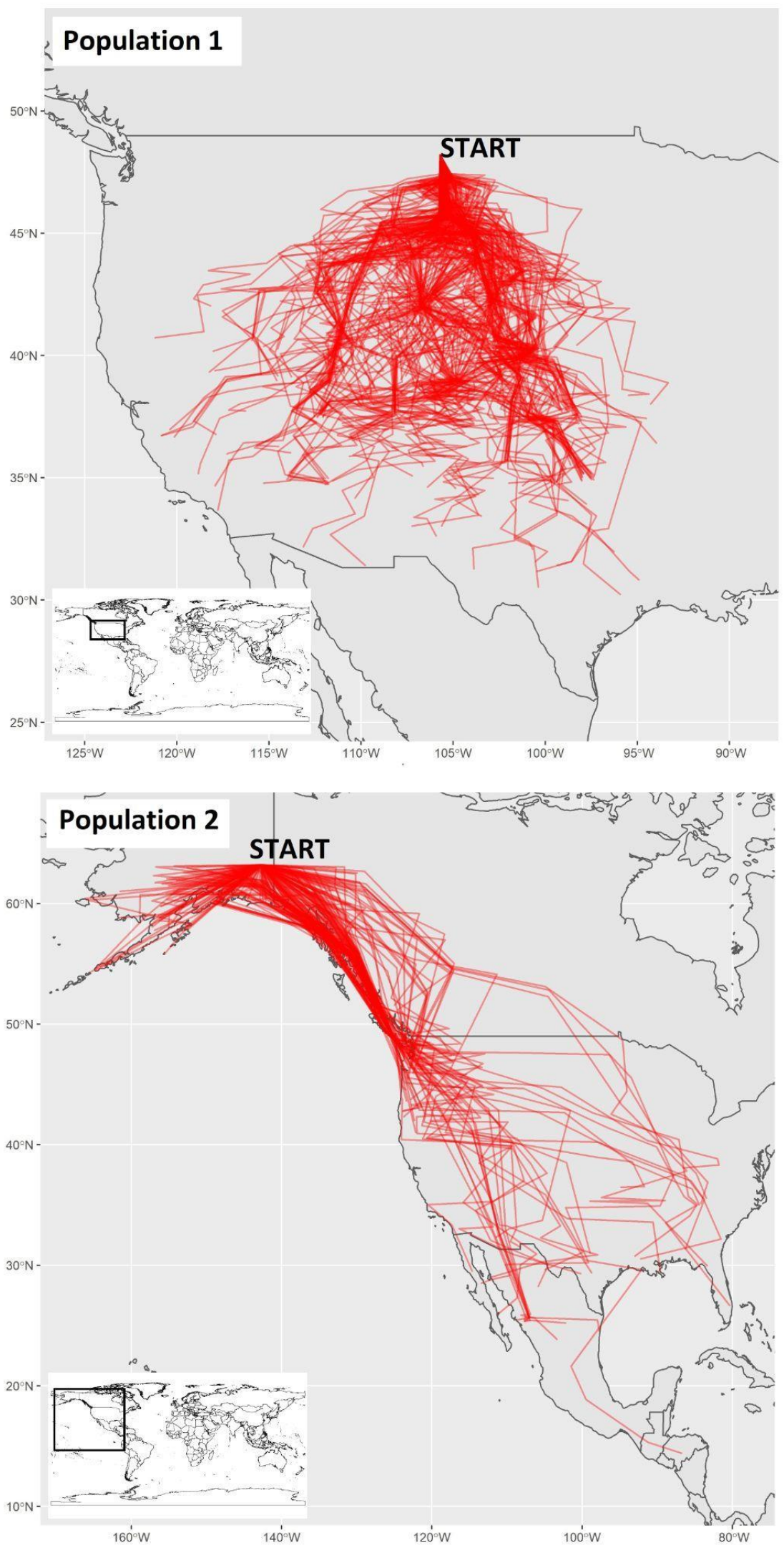
Figure 3. Movement tracks reveal that Population 1 tended to travel through the central United

States, while Population 2 traveled mostly throughout western Canada, United States, and Mexico.

297 Overall, Population 1 traveled more distance and exhibited more consistent paths near the origin than did Population 2. The movement tracks are natively produced by abmR. Inset world map provided for geographic reference.

While we can compare the movement tracks visually, Table 3 provides a numerical average remaining energy across all timesteps was 61.2 units compared to 99.7 units for P1.

There are several possible reasons for this observed pattern. First, P2 began with a smaller initial energy (70 units) than P1 (100 units). Additionally, P2 had higher optimum NDVI values $(0.6-0.8)$, which might have been less abundant and generally more difficult to reach encountered different raster cells along their journey.

\begin{tabular}{|c|c|c|c|c|c|c|}
\hline variable & mean & sd & median & $\min$ & $\max$ & range \\
\hline day & 7.4 & 4 & 7 & 1 & 14 & 13 \\
\hline $\bar{\equiv}$ longitude & -105.5 & 4.1 & -105.2 & -121.1 & -93.3 & 27.8 \\
\hline latitude & 41.6 & 3.4 & 41.9 & 30.2 & 47.5 & 17.3 \\
\hline energy & 99.7 & 1.3 & 100 & 80 & 100 & 20 \\
\hline delta energy & -0.04 & 1.5 & 0 & -20 & 20 & 40 \\
\hline distance & 154.6 & 70 & 154.4 & 5.5 & 316.7 & 311.1 \\
\hline day & 3.8 & 3.08 & 3 & 1 & 14 & 13 \\
\hline longitude & -126 & 17.3 & -127.1 & -166 & -80.3 & 85.7 \\
\hline latitude & 50.2 & 10.5 & 52.2 & 14.4 & 63.1 & 48.7 \\
\hline energy & 61.2 & 17.2 & 60 & 0 & 100 & 100 \\
\hline ¿ิ delta energy & -2.3 & 9.3 & -5 & -20 & 20 & 40 \\
\hline distance & 625.5 & 294 & 649.7 & 5.5 & 1445 & 1439.4 \\
\hline
\end{tabular}


311 Table 3. A numerical comparison of Populations 1 and 2, created by using values across all timesteps

312 for all agents. 'Day' summarizes the timestep variable of the movement tracks. 'Longitude' and

313 'latitude' summarize the geographical position of agents, while 'energy' summarizes agents'

314 remaining energy. 'Delta energy' corresponds to the change (gain or loss) of energy between each

315 timestep, while 'distance' refers to the distance traveled between each timestep. This table was

316 produced outside of abmR using raw movement data returned by the package.

317 Fig. 4 visually compares P1 and P2 movement outputs based on longitude and latitude. This

318 is not a native abmR figure, but rather is produced using the raw data that abmR generates to

319 show the flexible use of the package. In this figure, P1 movements tended to be to the east

320 and south of P2. However, P2 trajectory shows a much wider distribution, with density points

321 extending to the lower values of latitude. 

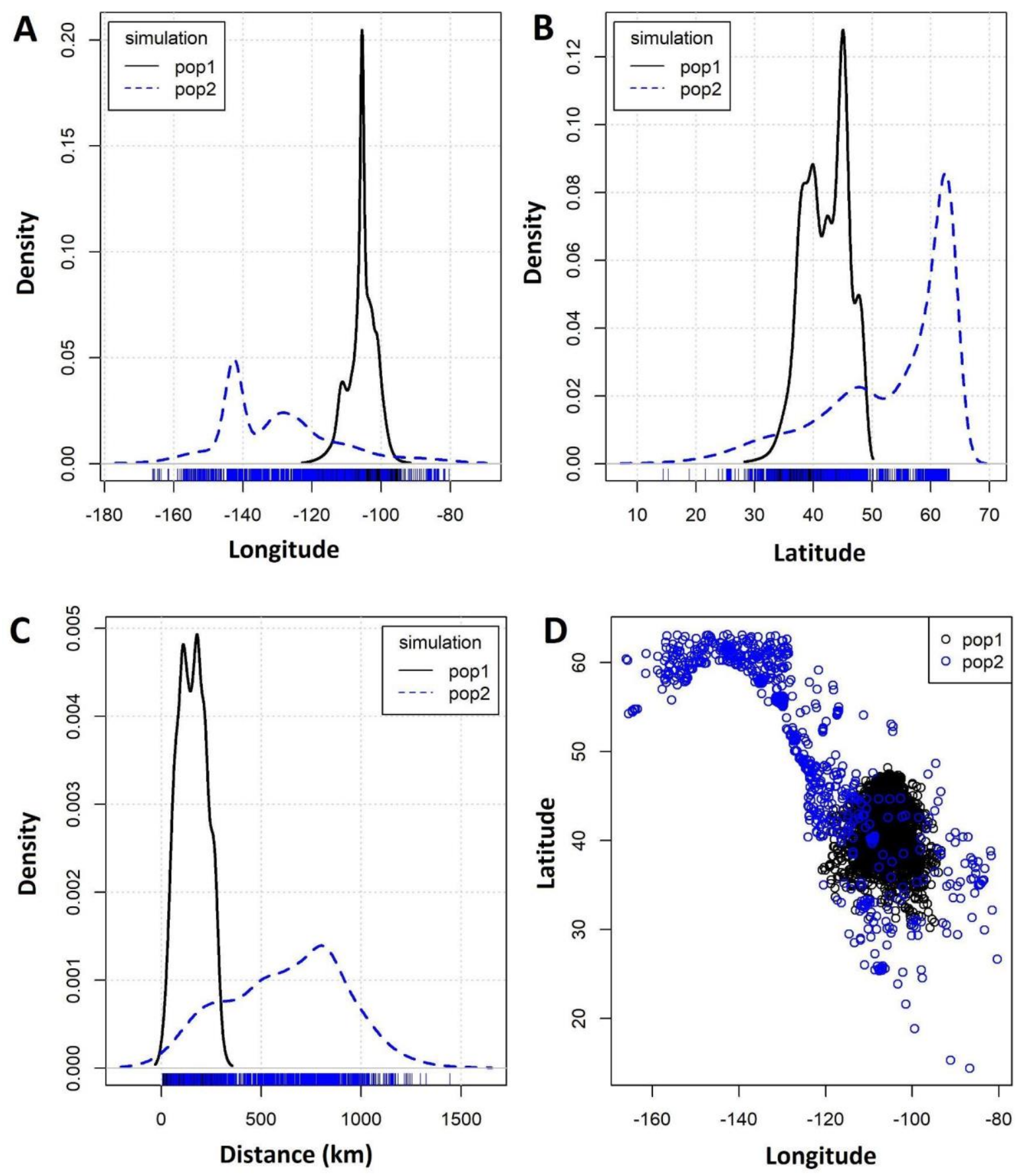

323 Figure 4. Graphical comparisons of Population 1 and Population 2 movements. Panels A and B show

324 density plots used to individually compare longitude (Panel A) and latitude (Panel B) coordinates

325 attained by agents from each population. Panel C compares the distance traveled between each

326 timestep, while Panel D shows geographical position for all agents in each population across all

327 timesteps. 
Finally, Fig. 5 provides a density surface plot for P2 describing agent energy gains (blue) and losses (red) across the landscape. This surface was created using the inverse distance weighted interpolation (IDW) function from the R package 'gstat' (Pebesma, 2004). IDW interpolates grid cell values across a surface using a linear combination of observed (sample) points. When interpolating a cell value, the value of the sample points closer to that cell carry a higher weight, while sample points further from that cell carry smaller weight. IDW is discussed in more detail in Wong (2017). The results from Fig. 5 match well with what we observe in Fig. 3. Movement tracks for P2 tend to follow the blue (energy gain) regions.

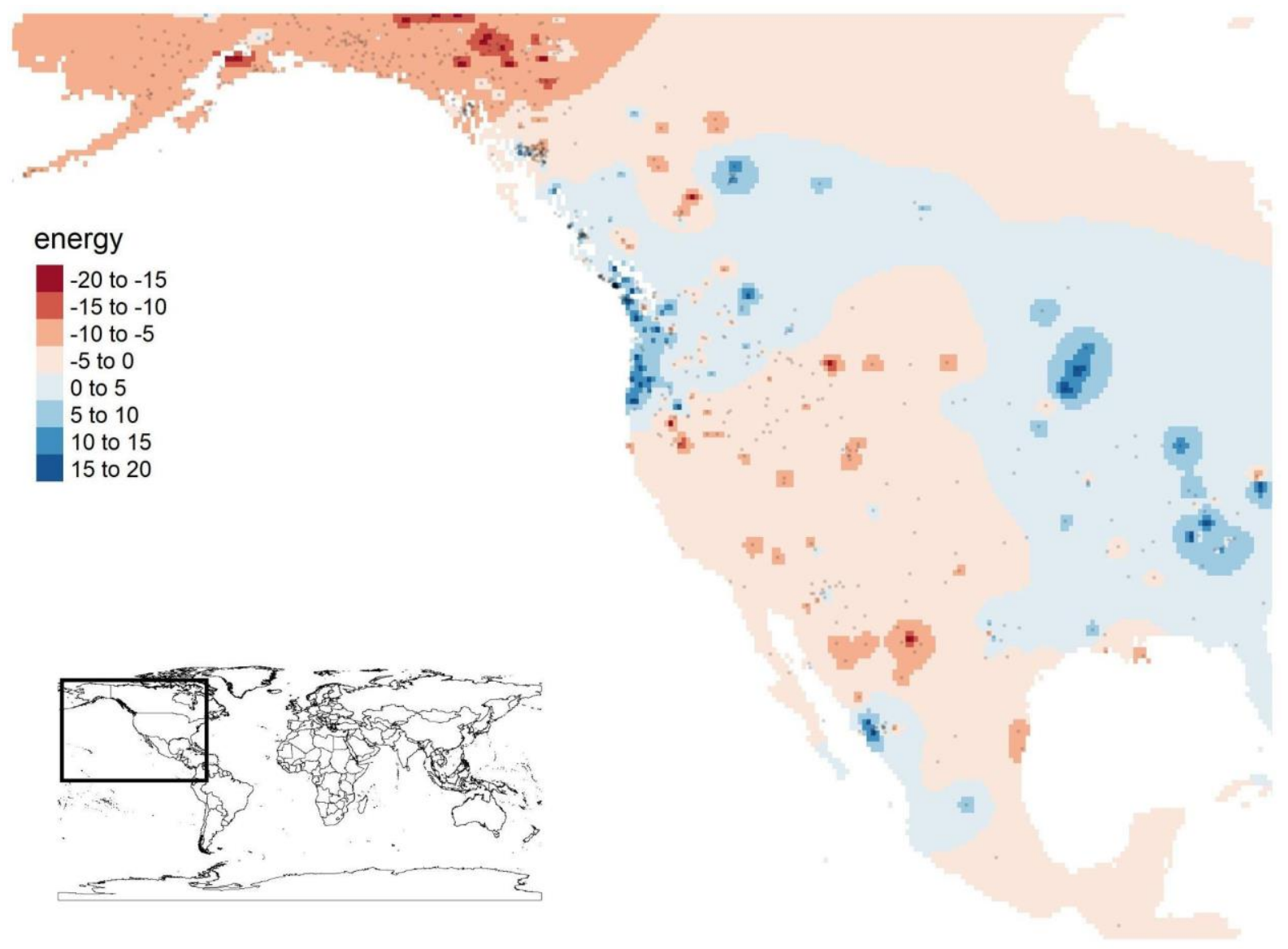

Figure 5. Energy gradient plot of Population 2 by timestep. Areas in red reflect energy loss (less suitable environmental values) while areas in blue reflect energy gain (better environmental values). This plot is produced directly by energyVI Z. Inset world map added for geographic reference. 


\section{CONCLUSIONS AND FUTURE WORK}

342

343

abmR provides a novel and efficient programming platform for simulating large-scale movements of species across taxa. We ran most of the initial test simulations on a local machine equipped with an Intel ${ }^{\circledR}$ Core $^{\mathrm{TM}}$ i7-5500U CPU $-2.40 \mathrm{GHz}$ and $8 \mathrm{~GB}$ of RAM and obtained results for 100-1000 agents within minutes. The novelty of the software includes the capability of concurrently modeling agent movement trajectories and energy budget. This feature enables a broader exploration of the ecological constraints that shape animal dispersal and/or migration. Moreover, abmR built-in arguments, such as fail_tresh, $n \_f a i l u r e s$, and energy_adj, provide additional flexibility when evaluating mortality scenarios that depend on baseline environmental conditions and energy requirement during prolonged movement bouts (see Table 2 for a full list of arguments affecting mortality).

Over the last decades, spatially explicit simulations and agent-based models have become more popular in ecological and evolutionary studies (Railsback et al., 2006; DeAngelis \& Grimm, 2014). Analytical platforms, such as InSTREAM, a simulation model approach designed to understand how stream and river salmonid populations respond to habitat alteration (Railsback et al., 2009), or ALMaSS, a predictive modeling tool for answering environmental policy questions regarding the effect of changing landscape structure on threatened animal species (Topping et al., 2003), allow investigation of specific ecological systems using ABM. On the other hand, many programming languages such as Netlogo, R, or Python are widely used to develop custom and more flexible models that can be adapted to address complex ecological or evolutionary research scenarios (Lustig et al., 2019; Chubaty $\&$ McIntire, 2021). However, the use of a programming language to develop a flexible ABM from scratch has two important drawbacks. First, it requires advanced programming skills. Second, its reproducibility can be compromised by the idiosyncrasies of the simulation 
algorithm written by the user. These idiosyncrasies, especially if not well documented, can make it difficult or even impossible for other researchers to replicate findings or adapt code to suit their modeling scenarios. abmR provides a novel framework to perform complex movement simulations through standardized functions and arguments that facilitate model annotation and reproducibility while providing publication-ready visualizations at the end of each run.

While we developed and tested abmR as a movement and energy budget simulation tool, its core software functionalities can be adapted to explore other processes such as disease outbreak scenarios (Dougherty et al., 2018). As an example, pathogen vector movement can be easily simulated within abmR, allowing the study of areas of confluence where disease transmission is more probable (Manore et al., 2015). Moreover, potential future updates include the ability to specify multiple raster stacks of different movement predictors and different species traits affecting the movement patterns in the function as. species.

Additionally, other code expansions might be useful to study plant seed dispersal, interactions of agents (density-dependent scenarios), and altitudinal movements.

\section{Acknowledgements}

We thank anonymous reviewers who provided invaluable feedback on an earlier draft. abmR relies heavily on a large set of R packages and we thank the $\mathrm{R}$ community for providing support and open-source code.

\section{Author Contributions}

B.G. led the development of the package and contributed extensively to the writing of the manuscript; J.F.L. contributed to the package, writing, and critical review of the manuscript; 
A.C. conceived the manuscript, led its writing, contributed to the package, and performed

388 package testing.

\section{Conflict of Interest Statement}

390 The authors declare that there are no financial or non-financial conflicts of interest.

\section{Data Availability}

$392 \mathrm{abmR}$ and the simulation results for Populations 1 and 2 are available for download from

393 Github at https://github.com/bgoch5/abmR. The environmental raster data used in the

394 examples is available at https://www.ncei.noaa.gov.

\section{Funding}

396 This study was supported in part by research grants from the U.S. National Science

397 Foundation (grants nos. EF 1840230 and DGE 1545261 and DEB 1911955), the National

398 Natural Science Foundation of China (81961128002), and by the Corix Plains Institute. 


\section{References}

Alerstam, T. (1993). Bird migration. Cambridge University Press.

Araujo, M. B. \& Guisan, A. (2006). Five (or so) challenges for species distribution modelling. J. Biogeogr. 33: 1677-1688.

Aurbach, A., Schmid, B., Liechti, F., Chokani, N., \& Abhari, R. (2020). Simulation of broad front bird migration across Western Europe. Ecological Modelling, 415, 108879.

Berg, H. C. (1983). Random Walks in Biology. Princeton Univ. Press, Princeton, NJ.

Bridge, E.S., Ross, J.D., Contina, A.J., \& Kelly, J.F., 2017. Using Agent-Based Models to Scale from Individuals to Populations. In Aeroecology (pp. 259-275). Springer, Cham.

Brown, D. G., \& Robinson, D. T. (2006). Effects of heterogeneity in residential preferences on an agent-based model of urban sprawl. Ecology and society, 11(1).

Chubaty A. M., \& McIntire E.J.B. (2021). SpaDES: Develop and Run Spatially Explicit Discrete Event Simulation Models. R package version 2.0.7. https://CRAN.Rproject.org/package $=$ SpaDES

Cushman, S. A., \& Lewis, J. S. (2010). Movement behavior explains genetic differentiation in American black bears. Landscape ecology, 25(10), 1613-1625.

DeAngelis, D. L., \& Grimm, V. (2014). Individual-based models in ecology after four decades. F1000prime reports, 6.

Dingle, H. (2014). Migration: the biology of life on the move. Oxford University Press, USA.

Dodge, S., Bohrer, G., Bildstein, K., Davidson, S.C., Weinzierl, R., Bechard, M.J., Barber, D., Kays, R., Brandes, D., Han, J., \& Wikelski, M. (2014). Environmental drivers of variability in the movement ecology of turkey vultures (Cathartes aura) in North and South America. Philosophical Transactions of the Royal Society B: Biological Sciences, 369(1643), p.20130195.

Dougherty, E.R., Seidel, D.P., Carlson, C.J., Spiegel, O., \& Getz, W.M., 2018. Going through the motions: incorporating movement analyses into disease research. Ecology letters, 21(4), pp.588-604.

Giuggioli, L., \& Bartumeus, F. (2010). Animal movement, search strategies and behavioural ecology: a cross-disciplinary way forward. Journal of Animal Ecology, 79(4), 906-909.

Goldstein, E., Erinjery, J. J., Martin, G., Kasturiratne, A., Ediriweera, D. S., de Silva, H. J., ... \& Iwamura, T. (2021). Integrating human behavior and snake ecology with agent-based models to predict snakebite in high risk landscapes. PLoS neglected tropical diseases, 15(1), e0009047. 
441 Grimm, V., Revilla, E., Berger, U., Jeltsch, F., Mooij, W. M., Railsback, S. F., ... \& from ecology. science, 310(5750), 987-991.

Grimm, V., \& Railsback, S. F. (2013). Individual-based modeling and ecology. Princeton university press.

Hawkes, C. (2009). Linking movement behaviour, dispersal and population processes: is individual variation a key?. Journal of Animal Ecology, 78(5), 894-906.

Hedenström, A. (2008). Adaptations to migration in birds: behavioural strategies, morphology and scaling effects. Philosophical Transactions of the Royal Society B: Biological Sciences, 363(1490), pp.287-299.

Holdo, R. M., \& Roach, R. R. (2013). Inferring animal population distributions from individual tracking data: theoretical insights and potential pitfalls. Journal of Animal Ecology, 82(1), 175-181.

Klimek, P., Poledna, S., Farmer, J. D., \& Thurner, S. (2015). To bail-out or to bail-in? Answers from an agent-based model. Journal of Economic Dynamics and Control, 50, 144154.

Lustig, A., James, A., Anderson, D., \& Plank, M. (2019). Pest control at a regional scale: Identifying key criteria using a spatially explicit, agent-based model. Journal of Applied Ecology, 56(7), 1515-1527.

Manore, C.A., Hickmann, K.S., Hyman, J.M., Foppa, I.M., Davis, J.K., Wesson, D.M., \& Mores, C.N. (2015). A network-patch methodology for adapting agent-based models for directly transmitted disease to mosquito-borne disease. Journal of biological dynamics, 9(1), pp.52-72.

Nathan, R., Getz, W. M., Revilla, E., Holyoak, M., Kadmon, R., Saltz, D., \& Smouse, P. E. (2008). A movement ecology paradigm for unifying organismal movement research. Proceedings of the National Academy of Sciences, 105(49), 19052-19059.

Pebesma, E.J. (2004). Multivariable geostatistics in S: the gstat package. Computers \& 468 Geosciences, 30: 683-691.

469 Polhill, J. G., Parker, D., Brown, D., \& Grimm, V. (2008). Using the ODD protocol for 470 describing three agent-based social simulation models of land-use change. Journal of 471 Artificial Societies and Social Simulation, 11(2), 3.

472 Railsback, S. F., Lytinen, S. L., \& Jackson, S. K. (2006). Agent-based simulation platforms: 473 Review and development recommendations. Simulation, 82(9), 609-623.

474 Railsback, S. F., Harvey, B. C., Jackson, S. K., \& Lamberson, R. H. (2009). InSTREAM: the 
Tang, W., \& Bennett, D.A. (2010). Agent-based modeling of animal movement: a review.

479 Geography Compass, 4(7), pp.682-700.

480 Thiele, J. C., Kurth, W., \& Grimm, V. (2012). RNetLogo: An R package for running and 481 exploring individual-based models implemented in NetLogo. Methods in Ecology and 482 Evolution, 3(3), 480-483.

483 Topping, C. J., Hansen, T. S., Jensen, T. S., Jepsen, J. U., Nikolajsen, F., \& Odderskær, P. 484 (2003). ALMaSS, an agent-based model for animals in temperate European landscapes. 485 Ecological Modelling, 167(1-2), 65-82.

486 Uhlenbeck, G. E., \& Ornstein, L. S. (1930). On the theory of the Brownian motion. Physical 487 review, 36(5), 823

488 Vermote, Eric; NOAA CDR Program. (2019): NOAA Climate Data Record (CDR) of 489 AVHRR Normalized Difference Vegetation Index (NDVI), Version 5. NOAA National 490 Centers for Environmental Information. https://doi.org/10.7289/V5ZG6QH9.

491 Willem, L., Verelst, F., Bilcke, J., Hens, N., \& Beutels, P. (2017). Lessons from a decade of 492 individual-based models for infectious disease transmission: a systematic review (2006493 2015). BMC infectious diseases, 17(1), 1-16.

494 Williams, H. J., Taylor, L. A., Benhamou, S., Bijleveld, A. I., Clay, T. A., de Grissac, S., ... $495 \&$ Börger, L. (2020). Optimizing the use of biologgers for movement ecology research. 496 Journal of Animal Ecology, 89(1), 186-206.

497 Wong, D. W. (2016). Interpolation: Inverse-Distance Weighting. International Encyclopedia 498 of Geography: People, the Earth, Environment and Technology: People, the Earth, 499 Environment and Technology, 1-7. 